\title{
Mucormicosis cutánea extensa: reporte de caso
}

\author{
Extensive cutaneous mucormycosis: a case report
}

\author{
Alvaro Vásquez-Palomino ${ }^{1, a ; 2, b}$ iD, Débora R. Mananita-Terrones ${ }^{1, a, b}$ iD, César Chian-García ${ }^{3, a}$ iD, \\ Eddie Angles-Yanqui ${ }^{1, a ; 4, b}$ iD
}

\section{RESUMEN}

La mucormicosis es una enfermedad oportunista causada por un grupo de hongos filamentosos del subfilo Mucormycotina, tiene una presentación clínica variada: rino-orbito-cerebral, pulmonar, cutánea, gastrointestinal, diseminada y de sitios poco frecuentes. La mucormicosis cutánea se presenta en el 19\% de los casos. Se presenta un caso de mucormicosis cutánea extensa en un paciente adulto joven, luego de quemadura por sustancia desconocida y aplicación de emplastos medicinales herbales. Tras recibir curaciones en varias oportunidades en un establecimiento de salud fue referido a un hospital de nivel 3 donde se le realizó limpieza quirúrgica y administración de antibióticos por vía endovenosa, sin mejoría clínica. Se le realizó estudio histológico de la piel afectada, cuyo informe fue inflamación intensa mixta a nivel del tejido subcutáneo, con moderado predominio de granulocitos, focos de necrosis grasa en parches, con variable inflamación. En el interior de los adipocitos necróticos, se observaron algunas estructuras con morfología de hifas fúngicas anchas, de contorno irregular morfológicamente consistentes con mucor. El paciente recibió antifúngicos combinados con curas quirúrgicas del tejido afectado en varias oportunidades, sin detener progresión de la infección. El paciente falleció.

PALABRAS CLAVE: Mucormicosis, zigomicosis, antifúngicos. (Fuente: DeCS BIREME).

\section{SUMMARY}

Mucormycosis is an opportunistic infection caused by a group of filamentous fungi belonging to the subphylum Mucormycotina. The clinical presentation is diverse including rhino-cerebral, pulmonary, cutaneous, gastrointestinal and disseminated locations. Cutaneous mucormycosis account for $19 \%$ of all cases. We report the case of a young male presenting with extensive cutaneous involvement after a burn with an unknown substance followed by local application of herbal medicines. He was initially treated in a peripheral health care center and was subsequently transferred to our hospital, where a surgical debridement was performed plus intravenous antimicrobial therapy with no improvement. A skin biopsy was performed showing fat necrosis and inflammation with granulocyte predominance. Broad hyphae were observed inside necrotic adipocytes characteristic of mucormycosis. The patient received parenteral antifungals plus surgical debridement but unfortunately died.

KEYWORDS: Mucormycosis, zygomycosis, antifungal agents. (Source: MeSH NLM).

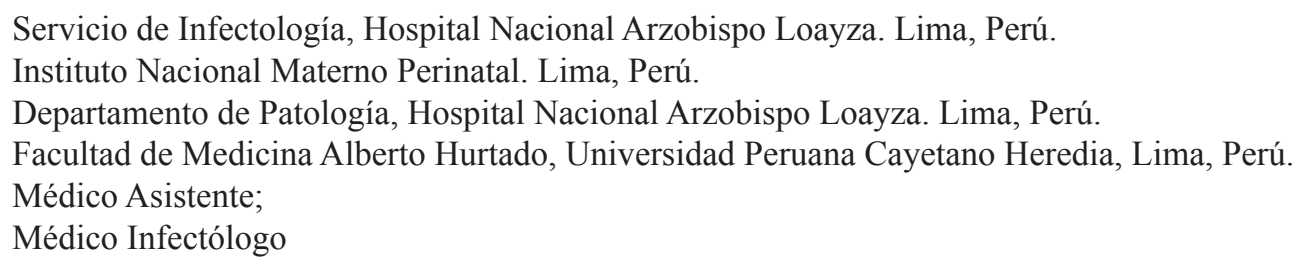




\section{INTRODUCCIÓN}

La mucormicosis cutánea resulta de la inoculación directa de esporas de zigomicetos en la piel, cuyo factor condicionante más importante es una lesión penetrante, afecta principalmente a huéspedes inmunocomprometidos y tiene alta mortalidad ${ }^{(1)}$. La mucormicosis cutánea se presenta en el $19 \%$ de los casos (2); en inmunocompetentes entre 43 y $67 \%$, el principal factor predisponente es un trauma penetrante (23-83\%) y está asociado a vendajes contaminados (8$15 \%{ }^{(3)}$. Las manifestaciones clínicas son variables, el inicio puede ser gradual, puede progresar lentamente o ser fulminante; la presentación típica es una escara necrótica acompañada de eritema circundante y endurecimiento ${ }^{(4)}$

La forma cutánea se puede clasificar como: primaria, cuando la infección es por inoculación directa o secundaria, por diseminación desde otros lugares ${ }^{(5)}$. Según su extensión se puede clasificar como a) Localizada (32 - 56\%), involucra tejido cutáneo y celular subcutáneo. b) Extensión profunda (24 - 52\%) con invasión a músculos, huesos y tendones, y c) Diseminada $(16-20 \%)^{(3)}$. La identificación temprana del hongo es esencial para establecer el manejo antifúngico y quirúrgico ${ }^{(5)}$.
En las infecciones de la piel, las infecciones fúngicas no están dentro de los primeros diagnósticos diferenciales, lo cual retrasa el diagnóstico y hace complejo su manejo.

La justificación de este reporte de caso radica en mostrar una de las formas inusuales de presentación clínica de la mucormicosis cutánea, a tener en cuenta por los clínicos para la sospecha diagnostica y planteamiento del manejo de forma oportuna en pacientes con esta afección.

El objetivo fue reportar un caso de una infección fúngica de presentación cutánea extensa, luego de quemadura por sustancia desconocida y aplicación de emplastos medicinales.

\section{PRESENTACIÓN DEL CASO}

Varón de 36 años de edad procedente del distrito de Huanta, Ayacucho, Perú, sin antecedente patológico previo. Refirió que tuvo una riña con un grupo de personas, quienes tras golpearlo le rosearon con una sustancia desconocida en la zona escapular derecha, ocasionándole una úlcera; un familiar le realizó curaciones con emplastos (hierbas medicinales) en varias oportunidades, sin presentar mejoría.
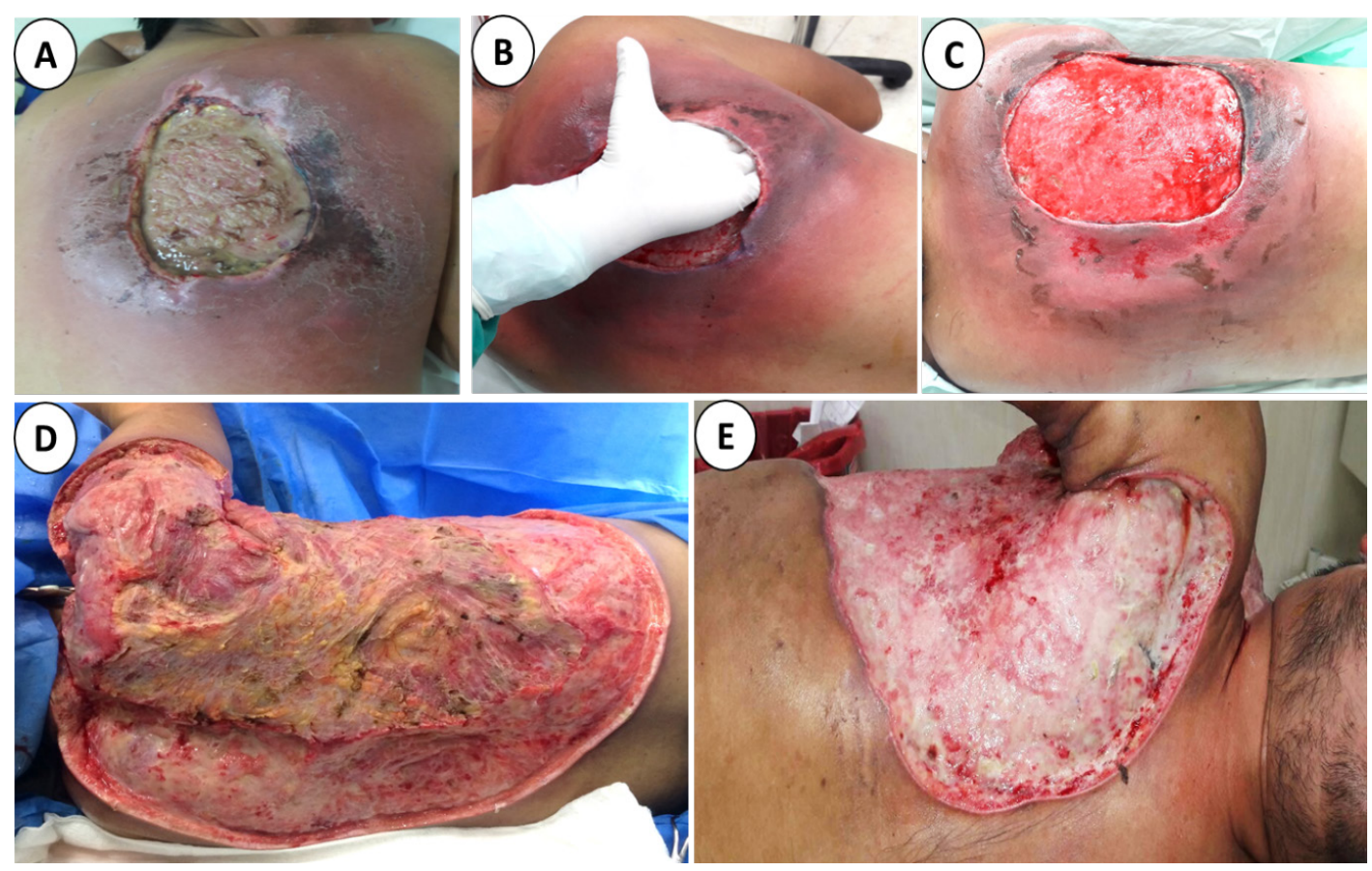

Figura 1. Mucormicosis cutánea extensa. A. Al momento de ingreso al hospital de tercer nivel. B. En la primera intervención quirúrgica. C. A los 19 días de hospitalización D. En el día 44 de hospitalización (zona posterior cervicotoracolumbar). E. En el día 44 de hospitalización (zona anterior axilar y pectoral). 
Posteriormente, fue atendido en un establecimiento de salud de la zona en donde le realizaron curaciones diarias y le indicaron antibióticos vía oral. Al no encontrar mejoría, luego de 9 días del incidente, con empeoramiento de la lesión, fue referido a un hospital de tercer nivel.

Al ingreso al hospital, presentaba una úlcera escapular de aproximadamente $15 \times 15 \mathrm{~cm}$, con secreción purulenta, bordes desvitalizados y signos extensos de flogosis perilesional (figura 1A), realizándose desbridamiento con posteriores curaciones diarias. En la evolución se evidencia empeoramiento del cuadro clínico por extensión de la lesión con zonas necróticas (figuras 1B y C). En el hemocultivo y cultivo de tejido de la lesión se aisló Acinetobacter baumannii multirresistente, por lo cual se administró ampicilina/sulbactam en combinación con meropenem.

A pesar de la cobertura antibiótica, la lesión se extendió a casi todo el dorso; se le realizó nuevo desbridamiento donde se evidenció tejido subcutáneo con signos de saponificación, secreción purulenta, signos de necrosis y abundante sangrado post procedimiento (figura 1A). Se tomó biopsia de tejido del centro de la úlcera y de tejido subcutáneo, se envía para cultivo y estudio anatomopatológico. El cultivo fue negativo y estudio de anatomía patológica consistente con Mucormicosis. Se inició terapia combinada con antifúngicos (anfotericina $\mathrm{B}$ deoxicolato más caspofungina) y limpiezas quirúrgicas por extensión de la lesión a tórax anterior (figura 1C, D y E); luego de varios días de hospitalizado, el paciente falleció.

\section{Reporte anatomopatológico:}

"Se recibió un fragmento de piel de 21 x $8 \mathrm{~cm}$. por $2,5 \mathrm{~cm}$. de profundidad, de aspecto congestivo, se observa a nivel de dermis y tejido subcutáneo áreas de aspecto necrótico y hemorrágico, a los cortes se evidencia tejido de aspecto graso con focos hemorrágicos. Al examen microscópico se observó inflamación intensa mixta a nivel del tejido subcutáneo (predominantemente septal), con moderado predominio de granulocitos. Focos de necrosis grasa en parches, con variable inflamación. En el interior de los adipocitos necróticos, algunas estructuras con morfología de hifas fúngicas anchas, de contorno irregular morfológicamente consistentes con mucor" (figura 2).

\section{DISCUSIÓN}

La mucormicosis es una enfermedad poco frecuente, difícil de diagnosticar con alta morbilidad y mortalidad. El diagnóstico a menudo se retrasa y la enfermedad tiende a progresar rápidamente. Son pocos los reportes de casos de mucormicosis cutáneas extensas y no se puede asegurar que una de las causas sea la aplicación de emplastos de hierbas medicinales ${ }^{(6)}$.

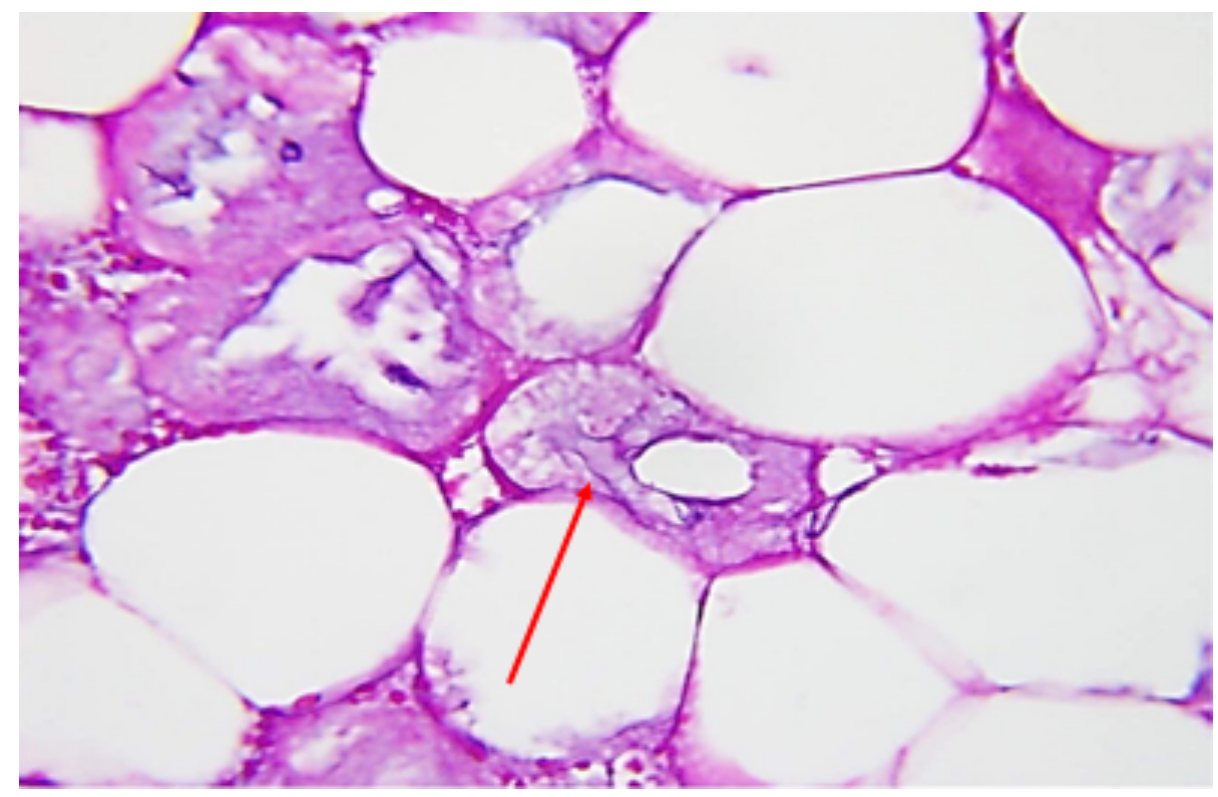

Figura 2. Estudio histopatológico: Hifa ancha ramificada (coloración PAS x 200X). 
En una revisión de 929 casos, 176 pacientes presentaron afectación cutánea. En la mayoría, la afección se mantuvo localizada en todo el tejido cutáneo, en el $24 \%$ alcanzó el tejido óseo y muscular, y en el $20 \%$ ocurrió diseminación hematógena a otros órganos no contiguos ${ }^{(2)}$.

La mayoría de los pacientes con mucormicosis cutánea tienen enfermedad de fondo como neoplasias hematológicas, diabetes mellitus o trasplante de órganos sólidos, pero existen reportes de mucormicosis cutánea y también como fascitis necrotizante en pacientes inmunocompetentes ${ }^{(7,8)}$. Según Roden et al. ${ }^{(2)}$, el 50\% de 176 pacientes y Skiada y Petrikkos ${ }^{(7)}$, en el $40 \%$ de 78 pacientes, eran inmunocompetentes. Prakash y Chakrabarti (3), mencionan que la presentación en inmunocompetentes varía de 43 a $67 \%$, siendo el principal factor predisponente el trauma penetrante (23-83\%) y está asociado a vendajes contaminados (8$15 \%)$.

La capacidad de diagnosticar mucormicosis depende del entrenamiento del personal de salud, así como contar con exámenes de imágenes, laboratorio, estudios histopatológicos y micológicos. La identificación a nivel de género y especie es recomendada para una mejor comprensión epidemiológica de la mucormicosis. Jeong et al. ${ }^{(9)}$, realizaron una revisión sistemática y metaanálisis de 851 reportes de casos de mucormicosis, en quienes para el diagnóstico se realizó el examen histopatológico en $83 \%$, de estos, en el $97 \%$ se reportaron hifas anchas, aceptadas o escasamente septadas; el cultivo se realizó en $69 \%$ de los casos, resultando $79 \%$ positivos para mucorales ${ }^{(9)}$. El tiempo medio hasta el diagnóstico, de datos disponibles de 199 casos, fue de 10 días después de la presentación del cuadro clínico.

La identificación molecular es fuertemente apoyada y preferida sobre la morfología; la mejor técnica para la identificación molecular es la secuenciación del espaciador transcrito interno, la identificación por matriz (MALDI-TOF) es moderadamente compatible $^{(10)}$.

En el caso que presentamos hubo demora en la sospecha de diagnóstico teniendo como un factor de confusión la exposición a sustancia química desconocida y una infección bacteriana extremadamente resistente.

Según las recomendaciones, la terapia de primera línea, requiere manejo multidisciplinario que incluye control de la condición de fondo, control de infecciones concomitantes, cirugía y terapia antifúngica, siendo los agentes más potentes in vitro anfotericina $\mathrm{B}$, posaconazol e isavuconazol (11). El antifúngico de elección es anfotericina $\mathrm{B}$ lisosomal como monoterapia (10,11). La combinación de antifúngicos como terapia de primera línea, fueron prometedores en las series de casos de pacientes; sin embargo, en un análisis de score de falla no mostraron beneficios ${ }^{(10)}$.

La combinación de antifúngicos se recomienda para terapia de salvataje, siendo las combinaciones, anfotericina B liposomal más caspofungina o posaconazol ${ }^{(11)}$. Se reportó éxito de tratamiento con isavuconazol, convirtiendo a este antifúngico en una opción terapéutica ${ }^{(12-14)}$, además de la recomendación de su uso en terapia de salvataje ${ }^{(10)}$.

Con relación a la mortalidad, en el estudio de Jeong et al. ${ }^{(9)}$, el promedio de mortalidad fue del $46 \%$, la más alta fue en pacientes con mucormicosis diseminada $(68 \%)$ y más baja en mucormicosis cutánea $(31 \%)$, diferenciando en esta última en cutánea localizada (31\%) y de extensión profunda (32\%).

Las lecciones de este caso, la mucormicosis cutánea en paciente adulto joven no es frecuente, es de presentación clínica inusual y se debe tener la sospecha en lesiones de piel previamente establecidas, expuestas a cualquier sustancia u objetos contaminados como vendajes, emplastos medicinales herbolarios, etc. y con pobre respuesta a antibióticos, además en lesiones con zonas necróticas por trombosis/isquemia, de tal modo que esta sospecha nos oriente hacia el diagnóstico temprano para brindar el tratamiento oportuno.

\section{Declaración de financiamiento y de conflictos de intereses:}

El reporte fue financiado por los autores. Los autores declaran no tener conflicto de intereses.

\section{Correspondencia:}

Alvaro Vásquez-Palomino

Correo electrónico: docteurpacifico@hotmail.com

\section{REFERENCIAS BIBLIOGRÁFICAS}

1. Reid G, Lynch JP, Fishbein MC, Clark NM. Mucormycosis. Semin Respir Crit Care Med. 2020; 41(1):99-114. 
2. Roden MM, Zaoutis TE, Buchanan WL, Knudsen TA, Sarkisova TA, Schaufele RL, et al. Epidemiology and outcome of zygomycosis: a review of 929 reported cases. Clin Infect Dis. 2005; 41(5):634-53.

3. Prakash H, Chakrabarti A. Global Epidemiology of Mucormycosis. J Fungi (Basel). 2019; 5(1):26. doi: 10.3390/jof5010026

4. Petrikkos G, Skiada A, Lortholary O, Roilides E, Walsh TJ, Kontoyiannis DP. Epidemiology and clinical manifestations of mucormycosis. Clin Infect Dis. 2012; 54 Suppl 1:S23-34.

5. Castrejón-Pérez AD, Welsh EC, Miranda I, OcampoCandiani J, Welsh O. Cutaneous mucormycosis. An Bras Dermatol. 2017; 92(3):304-11.

6. Ahmadinejad Z, Khazraiyan H, Ghanbari F, Ahmadi B, Gerami M. Cutaneous Mucormycosis in a Diabetic Patient following Traditional Dressing. Case Rep Dermatol Med. 2013; 2013:894927.

7. Skiada A, Petrikkos G. Cutaneous zygomycosis. Clin Microbiol Infect. 2009; 15 Suppl 5:41-5.

8. Jain D, Kumar Y, Vasishta RK, Rajesh L, Pattari SK, Chakrabarti A. Zygomycotic necrotizing fasciitis in immunocompetent patients: a series of 18 cases. Mod Pathol. 2006; 19(9):1221-6.

9. Jeong W, Keighley C, Wolfe R, et al. The epidemiology and clinical manifestations of mucormycosis: a systematic review and meta-analysis of case reports. Clin Microbiol Infect. 2019; 25(1):26-34.
10. Cornely OA, Alastruey-Izquierdo A, Arenz D, Chen SCA, Dannaoui E, Hochhegger B, et al. Global guideline for the diagnosis and management of mucormycosis: an initiative of the European Confederation of Medical Mycology in cooperation with the Mycoses Study Group Education and Research Consortium. Lancet Infect Dis. 2019; 19(12):e405-e421. doi: 10.1016/S1473-3099(19) 30312-3

11. Tissot F, Agrawal S, Pagano L, et al. ECIL-6 guidelines for the treatment of invasive candidiasis, aspergillosis and mucormycosis in leukemia and hematopoietic stem cell transplant patients. Haematologica. 2017; 102(3):433-44.

12. Zuglian G, Ripamonti D, Tebaldi A, Rizzi M. Cutaneous mucormycosis by Rhizopus arrhizus treated with isavuconazole as first line therapy: A case report. Med Mycol Case Rep. 2019; 26:42-3.

13. Marty FM, Cornely OA, Mullane KM, et al. Isavuconazole for treatment of invasive fungal diseases caused by more than one fungal species. Mycoses. 2018; 61(7):485-97.

14. Marty FM, Ostrosky-Zeichner L, Cornely OA, Mullane KM, Perfect JR, Thompson GR, et al. Isavuconazole treatment for mucormycosis: a singlearm open-label trial and case-control analysis. Lancet Infect Dis. 2016; 16(7):828-37.

Recibido: 22/03/2021 Aceptado: 23/09/2021 